\title{
Analysis of Neurite Outgrowth for a Laser Patterned Neuronal Culture
}

\author{
S. K. Guduru' ${ }^{1}$, S. V. Narasimhan ${ }^{2}$, S. T. Birchfield ${ }^{1}$, and B. Z. Gao ${ }^{2}$ \\ Department of ${ }^{1}$ Electrical and Computer Engineering and ${ }^{2}$ Bioengineering, Clemson University \\ Clemson, SC 29634 USA
}

\begin{abstract}
We describe a system for patterning neuronal cultures using a weakly focused laser beam. Using this technique, individual cells can be placed at predetermined positions with submicron accuracy, enabling detailed study of neurite outgrowths regulated by various cell contact interactions. Images of the cells are analyzed automatically by an algorithm to detect the soma and neurite outgrowth. The soma detection is based upon gray-level morphological processing, while the neurite outgrowth tracing is based upon the work of Al-Kohafi et al. [1], which has been modified to improve the computational performance. Results on images of embyonic chick forebrain neurons demonstrate the effectiveness of the technique.
\end{abstract}

\section{INTRODUCTION}

Manipulating individual neurons while maintaining cell physiology is a crucial part of constructing a biological neural network with specific design synapse connections. Such networks are important for studying neurite outgrowth, synapse formation, and neural control in normal and pathological conditions. The ability to coerce individual cells in these networks opens up a vast range of opportunities for neural engineering.

To this end, we have developed a patterned neuron culturing system that is capable of positioning individual cells at submicron accuracy. This system consists of a spatially resolved culture of 10 to 30 neurons, on which neurite outgrowth analysis is performed, surrounded by thousands of the same cells randomly deposited to provide conditioned media for the normal growth of the patterned neurons. The spatially resolved culture is created using a laser cell micropatterning system. This system allows for the creation of submicron-accurate patterns of cells onto substrates independent of substrate modification. An optical force generated by a weakly focused laser beam is used to trap and guide neurons onto a homogeneously coated substrate. Substrate translation allows for the creation of specific patterns, as shown in Fig. 1. To observe the neurite outgrowth and synapse formation under various patterning configurations, in this paper we concentrate on the development of an algorithm that automatically assesses the patterned cell culture under a microscope with an onstage incubator and a time lapse imaging facility. We describe a tracing algorithm based on that of Al-Kohafi et al [1] that uses Gaussian kernels of different sizes and orientations for neurite outgrowth analysis.

\section{NEURON PATtERNING}

\section{A. Laser Cell Micropatterning}

There are two conventional approaches to form a patterned in vitro cell culture. The first approach is to deposit the cells directly onto specific locations on the substrate. Cell printing is an example of this method through ink-jet printers [2] or laser forward transfer (MAPLE-DW) [3], both of which demonstrate great potential to create 3D tissue structures that can be used to create artificial tissue and even organs, if vascularization of the structures can be achieved. However, as micro cell patterning techniques, they are not feasible for the manipulation of single cells. As they are meant for creating macrostructures, their accuracy in creating microstructures is compromised. The second patterning method involves the modification of the surface properties and topology of the substrate, creating specific areas on the substrate that either promote or inhibit cell adhesion. Soft lithographic techniques, such as microcontact printing and microfluidics are examples of substrate patterning and are commonly used to pattern large numbers of cells at specific locations [4]. Although effective, these conventional surface patterning techniques may restrict normal cell spreading and neurite extension once the cells attach to the modified substrate. Therefore, they may not be suitable for research that requires natural cell migration driven by cell-cell interactions under a specific microenvironment. Furthermore, both substrate patterning and direct cell patterning are generally used to mimic the in vivo cell arrangement at a macro scale (large cell populations). Their spatial resolution is not high enough to study accurately cellcell interaction in a scale involving only a few cells, with complete control over intercellular contact and simultaneously allowing for normal cell migration to occur. Consequently, cellular arrangements created by conventional patterning techniques are not feasible for precise cell-cell contact interaction research.

Optical forces generated by a strongly focused laser beam are used to create a 3D trap of individual cells and other micro-sized dielectric particles in the focal point of the beam [5]. However, in a weakly focused laser beam, individual cells or dielectric particles proximal to the beam focus are drawn to the center of the beam and trapped within a 2D plane perpendicular to the beam axis. The trapped cells are then guided forward along the beam axis. Placement of a movable substrate perpendicular to the beam axis allows for the deposition of individual cells at specific positions on the substrate. Movement of the substrate, as the cells are trapped 


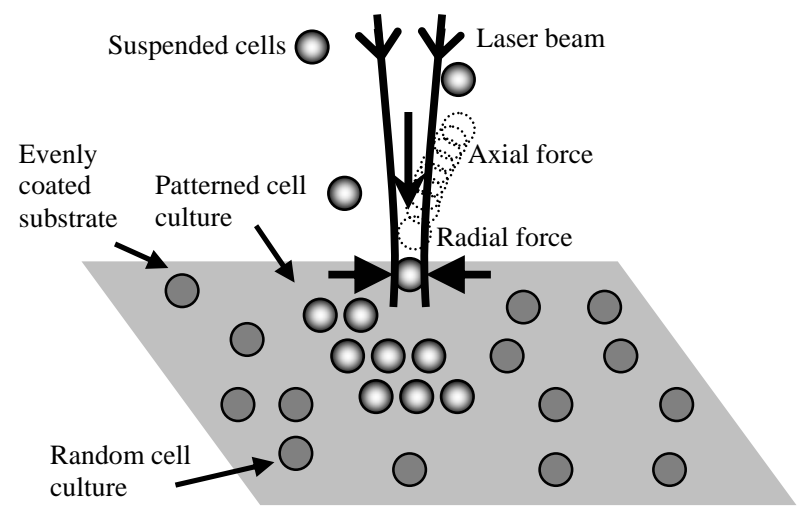

Fig. 1. Schematics of the laser cell micropatterning system.

and guided by the beam, allows for patterning cells in a process known as laser-guided direct-writing (LGDW) [6]. Cells patterned using LGDW maintain viability and normal cell function post pattern formation [7]. Using this technique, individual cells of different types can be picked out of a cell suspension and placed at any predetermined position with submicron accuracy. Pattern formation can take place on various substrates and also on substrates that contain an existing culture of cells [8].

\section{B. System Setup and Cell Culture}

A TEM $_{00}$ mode, tunable $(750 \mathrm{~nm}-950 \mathrm{~nm})$, continual wave, $\mathrm{Nd}: \mathrm{YVO}_{4}$ pumped solid state Ti:Sapphire laser (3900S, Spectra Physics CA) was expanded to the required numerical aperture (0.34) and focused into a custom-made laser cell deposition chamber. The chamber was mounted onto a threeaxis translation stage (FA-90, Aerotech, Pittsburgh), perpendicular to the axis of the beam. A $20 \mathrm{X}$ long working distance plan Mitutoyo objective (Edmund Optics, NJ) was placed under the substrate to view the pattern formation in real time. The objective was connected to a Sony XC-ST 70 CCD camera (Edmund Optics, NJ) through a C mount with IR filters to eliminate scattered laser beams. Illumination was added to the system through the use of a Dichroic mirror. A Matrox Meteor II frame grabber was used to transfer the images into the computer for analysis, and Video Savant 4 software (IO Industries, Ontario, Canada) was used to store the frames on the hard disk in real time (30 fps). Labview 7.0 from National Instruments was used for image processing purposes.

Day-7 embryonic chick forebrain neurons were harvested according to our protocol described previously [8] and the primary culture was used for the laser patterning. The neurons were grown on a substrate of gridded coverslips coated homogeneously with $0.1 \%$ poly-L-lysine hydrobromide (PLL; $\mathrm{Mw}=150,000$; Sigma; St. Louis, MO). A typical neuron pattern is shown in Fig. 2. Post pattern formation, the patterned cell culture was incubated in a $37{ }^{\circ} \mathrm{C}$ incubator with a $5 \% \mathrm{CO}_{2}$ atmosphere for 2 hours. After initial incubation, the created patterns were assessed using a Zeiss Axiovert 200M inverted microscope (Carl Zeiss, Thornwood, NY) with an

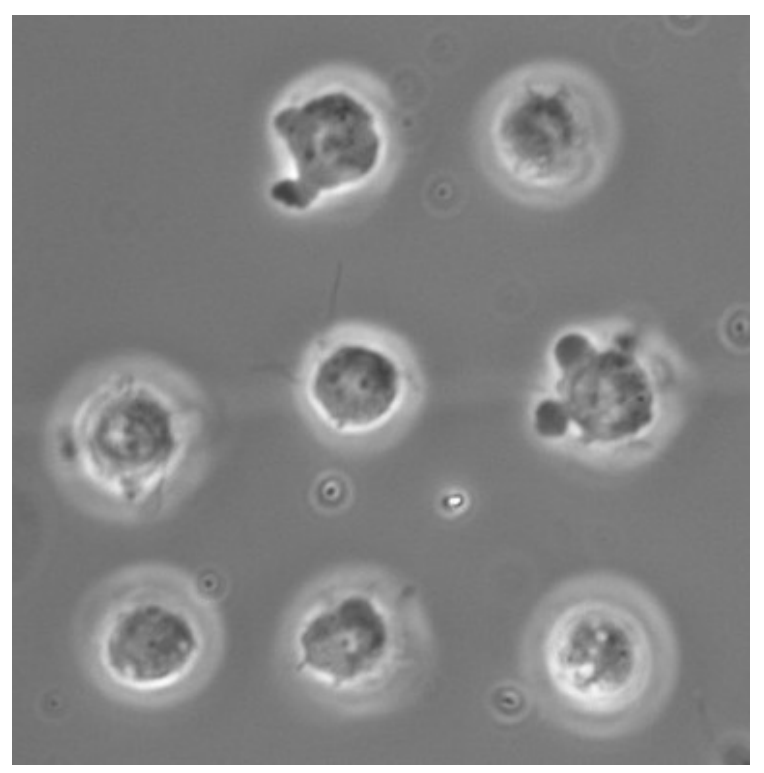

Fig. 2. A 3 by 3 neuron pattern (after the top left neuron migrated out of the field of view) with a spacing of $12 \mu \mathrm{m}$ ( $40 \mathrm{X}$ phase image).

onstage incubator. Time lapse images were acquired for the analysis of the neurite outgrowth and synapse formation.

\section{Automatic Neuron Detection and Tracing}

Our goal is to automatically process the images obtained by the microscope to locate the soma and neurite outgrowth. Broadly, two approaches exist for neurite tracing. The first approach uses adaptive filters and morphological operations such as thinning [9]. The second approach, which we adopt in this paper, is vectorial tracking in which a few initial points are located and then extended recursively based on local image properties. In this section we briefly describe the soma detection [10], followed by a more detailed description of the neurite tracing, which uses a modified version of the algorithm of presented by A. Can et al [11].

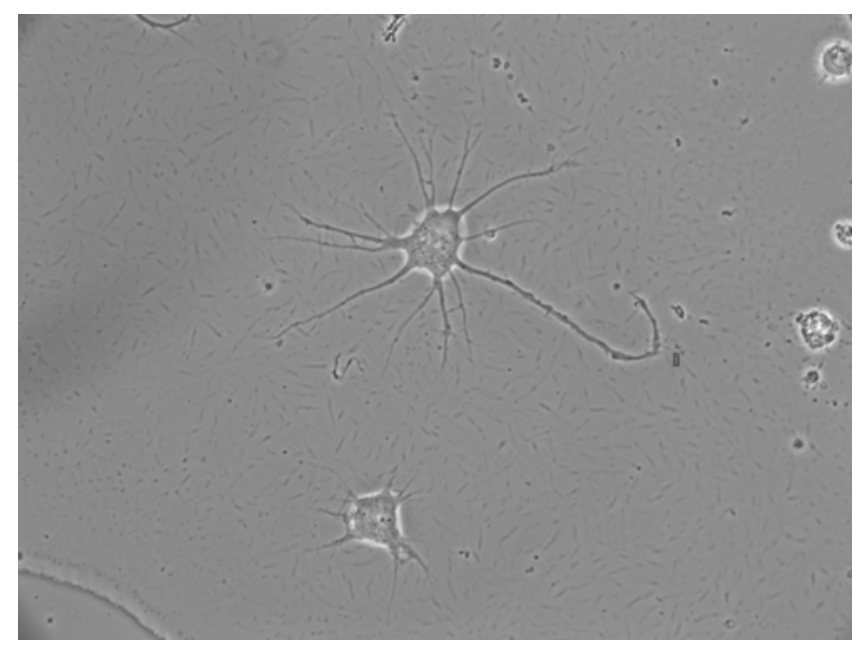

Fig. 3. Phase image ( $40 \mathrm{X}$ ) of a section of the patterned neurons. 


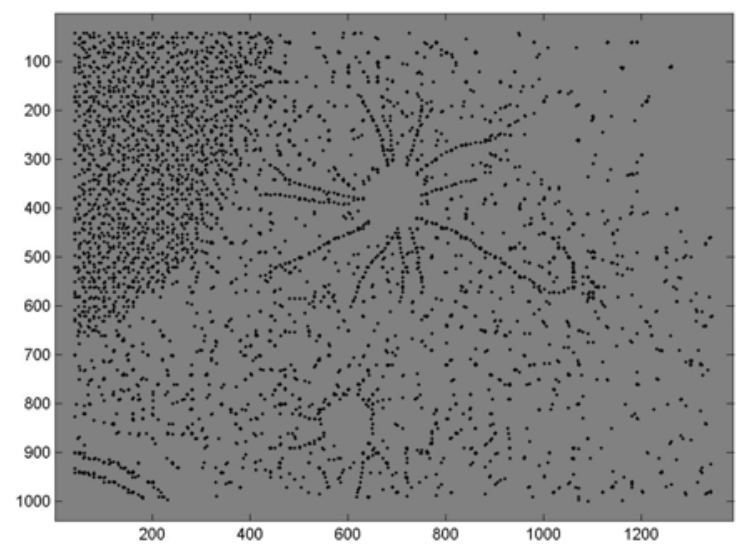

Fig. 4. Reliable seed points detected using normalized Gaussian kernels.

\section{A. Soma Detection}

The soma detection algorithm is straightforward and utilizes the fact that the size of the soma generally exceeds the neurite and noise. In our $1040 \times 1388$ images, the average radius of a soma is 50 pixels, and the average width of a neurite is 5 pixels. As a result, smoothing the image does not remove the item of interest. We apply grayscale morphological closing on the original image with a circular structuring element of radius 25 pixels [10]. Then the image is thresholded (using an adaptive algorithm that automatically determines the threshold so that $5 \%$ of the pixels are below it) to yield a binary image. Binary morphological opening is then applied using a circular structuring element with a radius of 15 pixels. Connected components are applied to the resulting binary image to yield to the individual soma. This simple technique works surprisingly well, detecting all the soma in the image shown in Fig. 6., with no false positives.

\section{B. Initialization of Seed Points}

Once the soma are detected, the neurite outgrowth is traced. The first step of the tracing is to initialize the seed points using the following procedure. For every $10^{\text {th }}$ row, a horizontal lowpass smoothing filter is applied. Then the pixels on the row are divided into non-overlapping groups of 10 pixels each. For each group, the pixel with the minimum value is selected as a seed point. This process is repeated for the columns in a similar manner, and all the seed points are combined together without distinction as to whether they have been detected by the row computation or the column computation. Note that by ignoring $90 \%$ of the data, the computation required by this step is greatly reduced, and yet a high density of seed points is found. Initial seed points in the soma detected regions are discarded.

\section{Selection of Reliable Seed Points}

Next a subset of the seed points are retained as reliable seed points, while the rest are discarded. Reliable seed points are determined by a two-step procedure. First, normalized Gaussian templates obtained by the outer product of a vertical Gaussian vector and a horizontal constant vector are applied to

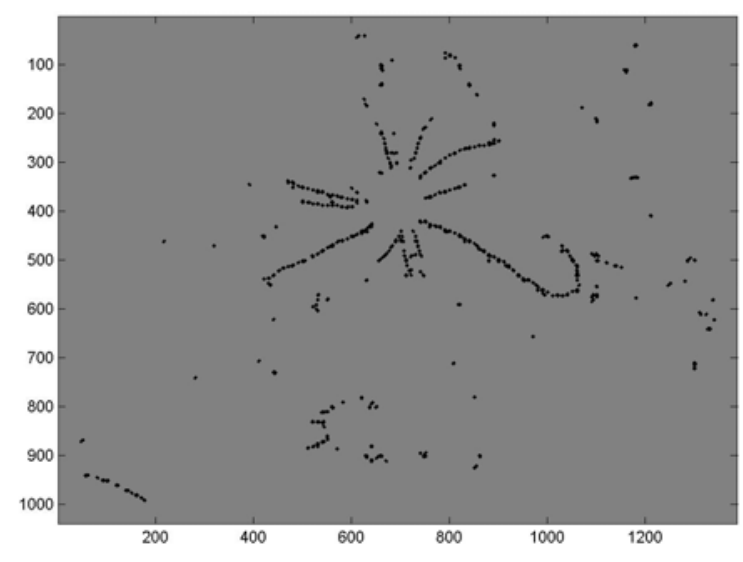

Fig. 5. Reliable seed points detected.

each seed point at 16 equally-spaced orientations and 3 scales $(9 \times 9,11 \times 11$, and $13 \times 13)$. The templates are multiplied by -1 because the neurites are darker than the background. Seed points that yield two maxima approximately 180 degrees apart, and for which both maxima exceed a threshold, are retained, while the rest are discarded.

The seed points thus retained are subjected to a second test based on the approach of A. Can et al. [11]. Derivative kernels are applied on either side of each seed point, using the direction found by the previous step as well as +/- one orientation (22.5 degrees). The kernels detect the presence of intensity edges on the left and right side of the neurite on which the seed point is located. Seed points for which both the left and the right edges exceed a threshold are retained as reliable seed points.

We have found the two-step procedure to be necessary for good performance. As shown in Figures 4 and 5, the second step removes a large number of false positives that arise in the first step due to the gradual intensity gradient in the upper-left corner of the image. Not shown in the figures is the fact that the second step, without the first, yields incorrect directions for many of the neurites because the derivative kernels are distracted by intensity variations within the neurites. Moreover, the computation involved in our two-step procedure is less than that of Can et al. [11], because the Gaussian kernels are faster to compute.

\section{Recursive Tracing of Neurites}

The next step is to recursively trace the neurites using the normalized Gaussian template. Each reliable seed point is traversed in the forward and backward directions by computing the score of the template at three different orientations (the current orientation as well as +/- 22.5 degrees) and three different scales (the current scale as well as $+/$ - one). If the maximum of these nine scores is greater than a threshold, then the neurite is extended from the current position along a fraction of the length of the template, and the procedure is continued.

These steps are repeated until one of the following stopping criteria is met: (1) the new point is outside the border 


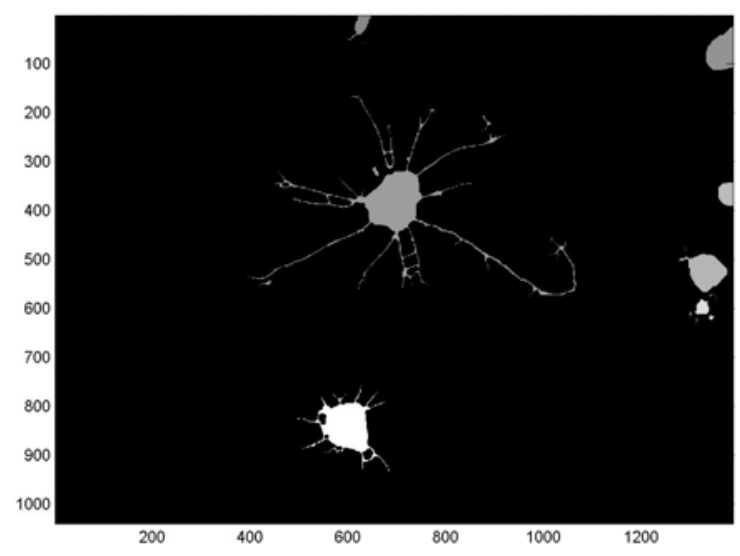

Fig. 6. Soma and neurites detected by the algorithm. A different gray level is assigned to each neuron.

of the image; (2) the new point is in the vicinity of a previously detected neurite or soma; or (3) the sum of the maximum template responses in opposite directions is less than a threshold. The corresponding result can be seen in Fig. 6.

\section{E. Labeling Neurons}

A binary two-dimensional array with one bit per pixel in the original image is used to keep track of the previously detected soma and neurites. All the values are initialized to be zero. Values are set to 1 wherever a soma is detected or when a neurite is traced. After tracing is completed, the connected components of the array are computed, yielding a preliminary labeling for each soma and neurite. Then an iterative process connects each neurite with a nearby soma, or a dendrite that has already been connected to a soma, within a $7 \times 7$ neighborhood. For connection purposes, the soma are dilated by 25 pixels to overcome the effect of the light band around the soma. After the process converges (six iterations for the image shown in Fig. 3), neurites that are not connected to any soma are discarded. All the retained neurites receive the label of the soma to which they are connected.

\section{CONCLUSIONS}

We have described a system to pattern neuronal cultures using a weakly focused laser beam. The system is capable of positioning individual cells at submicron accuracy, thus opening the door for more precise studying of neurite outgrowth, synapse formation, and neural control in normal and pathological conditions. We have also described an algorithm for automatically analyzing the images of the cells captured by our system.
The algorithm detects the soma using gray-level morphology, then it detects and traces the neurites using Gaussian kernels., followed by a labeling process that assigns a different label to neuron. On a noisy image from our system, the algorithm has proved effective at capturing all the neurons with all the neurites. Future work should be aimed at running the algorithm on a video sequence to measure the outgrowth of the neurites automatically.

\section{ACKNOWLEDGMENTS}

The authors would like to thank Mr. Daniel Bakken, Department of Bioengineering, Clemson University, for his valuable assistance in neuron culture and image acquisition. The project was supported partly by the South Carolina Spinal Cord Injury Fund and South Carolina BRIN Program.

\section{REFERENCES}

[1] K. A. Al-Kofahi, A. Can, S. Lasek, D. H. Szarowski, N. Dowell-Mesfin, W. Shain, J. T. Turner, and B. Roysam, "Median-based robust algorithms for tracing neurons from noisy confocal microscope images," IEEE Transactions on Information Technology in Biomedicine, vol. 7, pp. 302-317, 2003.

[2] T. Xu, S. Petridou, E. H. Lee, E. A. Roth, N. R. Vyavahare, J. J. Hickman, and T. Boland, "Construction of high-density bacterial colony arrays and patterns by the ink-jet method," Biotechnology and Bioengineering, vol. 85, pp. 29-33, 2004.

[3] P. K. Wu, B. R. Ringeisen, D. B. Krizman, C. G. Frondoza, M. Brooks, D. M. Bubb, R. C. Y. Auyeung, A. Pique, B. Spargo, R. A. McGill, and D. B. Chrisey, "Laser transfer of biomaterials: Matrix-assisted pulsed laser evaporation (MAPLE) and MAPLE Direct Write," Review of Scientific Instruments, vol. 74, pp. 2546-2557, 2003.

[4] R. S. Kane, S. Takayama, E. Ostuni, D. E. Ingber, and G. M. Whitesides, "Patterning proteins and cells using soft lithography," Biomaterials, vol. 20, pp. 2363-76, 1999.

[5] A. Ashkin, "History of optical trapping and manipulation of smallneutral particle, atoms, and molecules," IEEE Journal of Selected Topics in Quantum Electronics, vol. 6, pp. 841-856, 2000.

[6] D. J. Odde and M. J. Renn, "Laser-guided direct writing of living cells," Biotechnology and Bioengineering, vol. 67, pp. 312-318, 2000.

[7] S. V. Narasimhan, R. L. Goodwin, T. K. Borg, D. M. Dawson, and B. Z. Gao, "Multiple Beam Laser Cell Micro Patterning Technique," Proceedings of SPIE, vol. 5514 Optical Trapping and Optical Micromanipulation, pp. 437, 2004.

[8] D. Bakken, S. V. Narasimhan, K. Burg, and B. Z. Gao, "Laser Micropatterning of Polylactide Microspheres into Neuronal-Glial Coculture for the Study of Axonal Regeneration," Volumes of Macromolecular Symposia, (in press), 2005.

[9] A. Hoover, V. Kouznetsova, and M. Goldbaum, "Locating blood vessels in retinal images by piecewise threshold probing of a matched filter response," IEEE Transactions on Medical Imaging, vol. 19, pp. 203210, 2000.

[10] K. Al-Kofahi, L. Lasek, D. H. Szarowski, C. Pace, G. Nagy, J. N. Turner, and B. Roysam, "Rapid Automated three-dimensional tracing of neurons from confocal image stacks," IEEE Transactions on Information Technology in Biomedicine, Vol. 6, pp. 171-187, 2002.

[11] A. Can, H. Shen, J. N. Turner, H. L. Tanenbaucm, and B. Roysam, "Rapid automated tracing and feature extraction from retinal fundus images using direct exploratory algorithms," IEEE Transactions on Information Technology in Biomedicine, vol. 3, pp. 125-138, 1999. 